\title{
Frequency-specific cellular changes in the auditory system during acquisition and reversal of discriminative conditioning
}

\author{
JEAN-MARC EDELINE, N. NEUENSCHWANDER-EL MASSIOUI, and G. DUTRIEUX \\ Département de Psychophysiologie, Laboratoire de Physiologie Nerveuse \\ CNRS, Gif-sur-Yvette, France
}

\begin{abstract}
The possibility that learning induces frequency-specific changes at different levels of the auditory system was investigated by recording multiunit activity at the level of the auditory cortex, the magnocellular medial geniculate, and the dorsal cochlear nucleus. Chronically implanted rats were submitted to frequency discrimination (CS+/CS-) and reversal training while they were engaged in a leverpressing for food task. The conditioned suppression response indicated the acquisition of the discrimination and its reversal at the behavioral level. Off-line test sessions, during which different frequencies were tested, were conducted before conditioning, after acquisition of the initial discrimination, and after its reversal. They showed that frequency-specific changes occurred during the initial discrimination at the level of the auditory cortex, the medial geniculate, and, to a lesser extent, the dorsal cochlear nucleus. After acquisition of the reversal, frequency-specific changes, in relation to the new reinforcement rule, were also detected at the cortical and thalamic level but not at the cochlear nucleus level. These results indicate that frequency-specific changes can occur during conditioning at different levels of the CS auditory pathway and suggest that interactions between these different levels must be studied to understand modifications of information processing during learning.
\end{abstract}

Since the pioneering work of Buchwald, Halas, and Schramm (1966), many groups have reported that pairing an acoustic conditioned stimulus (CS) with an unconditioned stimulus (US) induces modifications of the sensory responses elicited by the CS in certain structures of the auditory system. The work of Olds, Disterhoft, Segal, Kornblith, and Hirsh (1972), Gabriel, Saltwick, and Miller (1975), Oleson, Ashe, and Weinberger (1975), Ryugo and Weinberger (1978), Disterhoft and Stuart (1976, 1977), and Birt and Olds (1981) has demonstrated the associative nature of these changes. The main problem of these results is that the changes occurring in sensory systems do not appear to be very different from the cellular conditioning occurring in many non-sensory structures of the brain. In fact, two explanations can be postulated for the occurrence of changes in sensory structures: (1) These changes have no more specificity than any cellular change occurring in other structures, thus raising the possibility that these sensory modifications are only a passive consequence of the associative changes that take place in more integrative structures. (2) These changes reflect

This work was supported by Fellowship 84158 from the Ministère de la Recherche et de la Technologie. The authors wish to thank E. Dubois-Hennevin, B. Hars, and A. Villa for their comments on the earlier version of this paper. Special thanks are due R. Lennartz for his useful comments and his help in improving the language. Correspondence should be addressed to Jean-Marc Edeline, Center for the Neurobiology of Learning and Memory, Bonney Center, University of California, Irvine, CA 92717. learning-induced modifications in the sensory encoding. In other words, the encoding of the physical dimensions of the CS (e.g., the frequency, the intensity, or the direction) is affected by learning. The data obtained by Diamond and Weinberger $(1986,1989)$, Bakin, Condon, and Weinberger (1988) bring strong arguments in favor of the latter hypothesis. The shifts of tuning curves that they observed after conditioning in the auditory cortex suggest that changes in tonal frequency processing take place in areas AII and VE. Since almost all the cortical neurons showed such changes, these researchers have proposed that learning induced a reorganization of the cortical tonotopy. They supposed that this phenomenon is possible by convergence, at the cortical level, of stable auditory information coming from the ventral MG (vMG) and of learning-induced changes coming from the medial $M G$ (mMG; see Weinberger et al., 1990, for review). Implicitly, this interpretation supposes that the conditioned changes occurring at the subcortical level do not have the same degree of selectivity as do those observed at the cortical level. As an example, the changes observed in the mMG could contribute to the cortical plasticity, but selective shifts of tuning curves are not supposed to occur at the thalamic level.

However, many studies have reported conditioned changes at different subcortical levels: in the $\mathrm{mMG}$ (Birt \& Olds, 1981; Disterhoft \& Stuart, 1976; Gabriel et al., 1975; Olds et al., 1972; Ryugo \& Weinberger, 1978), in some subdivisions of the inferior colliculus (Disterhoft \& Stuart, 1977), and even in the dorsal cochlear nucleus 
(DCN; Buchwald et al., 1966; Halas, Bearlsey, \& Sandlie, 1970; Oleson et al., 1975). Thus, the question can be raised as to whether or not the shifts of tuning curves observed by Weinberger and his colleagues are really a characteristic of the cortex, or whether or not such changes can be detected in subcortical structures. The ideal experiment to answer this question requires recording tuning curves simultaneously at different levels of the auditory pathway in order to determine in which structures shifts of tuning occur after conditioning. Such an experiment seems technically infeasible at this time, since this type of study is already quite difficult when only one structure is monitored. To address the question of the specificity of the changes occurring at different levels of the auditory system, a more simple approach is to test the multiunit changes detected at different levels in a generalization gradient (i.e., test responses to different frequencies, including the CS). No such attempt has been conducted in previous studies showing conditioned multiunit changes in the auditory system. The main problem of this approach is that the CS frequency is the same across animals and sites, even though the responses to this frequency may be very different at the different sites. To control for this, we decided to test the selectivity of conditioned changes in a generalization gradient during both a frequency discrimination and its reversal between a reinforced conditioned stimulus (CS + ) and a nonreinforced stimulus ( $\mathrm{CS}-$ ). In addition, the frequencies used as $\mathrm{CS}+$ and CS - were counterbalanced between animals. The idea of this design was that, if frequency-selective changes can be detected in a gradient generalization after both acquisition and the reversal of a discrimination independently of the frequency used as CS + and CS -, we can attribute these changes to learning and not to bias coming from the choice of the CS frequency.

Thus, in the present experiment, we compared the selectivity of the conditioned changes occurring in the same animals at the level of the auditory cortex (AC), the magnocellular medial geniculate (mMG), and the dorsal cochlear nucleus (DCN); we have tested this selectivity during discrimination and discrimination reversal. The conditioned suppression of leverpressing for food was used as a behavioral measure of learning during the acquisition of the initial discrimination and its reversal. Test sessions, during which generalization gradients were examined, were conducted off-line (without animal barpressing) in order to minimize the possible contamination of recordings by movements and masking noises. These test sessions took place after 40 discrimination trials so that we could compare our results with those of Weinberger and his colleagues, who have observed frequency-specific modifications of tuning curves in the AC after about 40 trials.

\section{METHOD}

\section{Subjects}

The subjects were 6 male Sprague-Dawley rats, weighing 290 to $330 \mathrm{~g}$ at the time of surgery. The electrodes were stereotaxically implanted while the animals were under deep anesthesia (pento- barbital, $50 \mathrm{mg} / \mathrm{kg}$ ). After surgery, the animals were placed in individual cages under a natural dark:light cycle. At least 1 week of recovery was allowed before the start of the experiment.

\section{Electrodes Implantation}

The implantation and recording procedures were the same as those used in a previous study (Edeline, Dutrieux, \& NeuenschwanderEl Massioui, 1988). Briefly, two electrodes (62- $\mu \mathrm{m}$-diameter Nichrome wires sharpened under microscope control, 250-900 kn at $150 \mathrm{~Hz})$ were inserted into a stainless steel microtube $(200 \mu \mathrm{m}$ internal diameter) whose noninsulated extremity was used for differential recording. The electrodes connected to three miniature sockets were slowly lowered independently for each brain area. The stereotaxic coordinates were derived from the Paxinos and Watson (1982) atlas. For each structure, the final adjustment of the electrodes was made to obtain the strongest responses to probe tones delivered via the hollow ear bars. A small silver sphere, inserted between the dura and the parietal bone, was used as a reference. This reference and the multichannel sockets were fixed to the skull with dental acrylic cement. During 3 days after surgery, the animal received an injection of antibiotics (Terramycine, $45 \mathrm{mg} / \mathrm{kg}$ ). After at least 1 week of recovery, each animal was progressively reduced to $85 \%$ of its individual ad-lib body weight.

\section{Apparatus}

The experimental cage $(23 \times 23 \times 47 \mathrm{~cm})$ was placed in a soundattenuating chamber, where an exhaust fan provided a background noise of $40 \mathrm{~dB}$. The chamber was equipped with a 2-W light at the top of the left wall and a loudspeaker (Siare, $6 \mathrm{~cm}$ in diameter, bandpass $20-20000 \mathrm{~Hz}$ ) on the rear wall. The four pure tone frequencies used in this study were generated by four different oscillators adjusted individually under oscilloscope control. For each frequency $(1,4,10,14 \mathrm{kHz})$, the intensity was adjusted to obtain $75 \mathrm{~dB}$ spl at the center of the cage (approximately at the height of the animal's head) as measured by a B\&K sound-level meter. The grid floor was made of stainless steel rods, $.5 \mathrm{~cm}$ in diameter and spaced $1.5 \mathrm{~cm}$ center to center. A stainless steel lever $(1.5 \times 3.8 \mathrm{~cm})$ and a food magazine could be placed on the right wall. Counterbalanced cables were connected to the animal; these cables emerged from the top of the cage through a multichannel rotating connector.

\section{Behavioral Training}

After 2 days of familiarization with the experimental cage and recording cables, the animals were trained to get food pellets from the magazine in a $30-\mathrm{min}$ session. The next day, the lever was available and allowed the animals to get continuous reinforcement until 50 leverpresses. During the 2 subsequent days, the animals were trained on a VI30 schedule (i.e., variable interval, mean inter$\mathrm{val}=30 \mathrm{sec}$ ) for two 30 -min sessions, followed by five sessions of VI60 of the same duration. For the next 2 days, two habituation sessions were carried out on the baseline of the instrumental training. In each of the sessions, 10 tones (each $10 \mathrm{sec}$ in duration) were presented in a pseudorandom order with a mean intertrial interval (ITI) of $3 \mathrm{~min}$ (range $=2-4 \mathrm{~min}$ ). Five of these tones were $1 \mathrm{kHz}$ and the other five were $14 \mathrm{kHz}$. Conditioning began the next day by pairing one of the two frequencies with an electrical footshock (. $3 \mathrm{~mA}, .5 \mathrm{sec}$ in duration). The reinforced stimulus $(\mathrm{CS}+)$ was the $1-\mathrm{kHz}$ tone for half of the animals and was the $14-\mathrm{kHz}$ tone for the other half. After six sessions (each $45 \mathrm{~min}$ in duration; five $\mathrm{CS}+$ and five CS - at each session) of this initial discrimination the reinforcement rule was reversed: for all of the animals, the frequency previously used as CS + became the nonreinforced stimulus (CS - ) and the previous CS - was followed by the electrical footshock. Four sessions of this reversed discrimination were conducted. During both the initial and the reversal discrimination the mean ITI was $3 \mathrm{~min}$ (range $=2-4 \mathrm{~min}$ ). 
The entire experiment (stimulus presentations and delivery of food pellets) was controlled by a microcomputer, which also recorded the behavioral data. On each trial, the leverpresses were counted during the $10 \mathrm{sec}$ of tone and during the $10 \mathrm{sec}$ preceding the tone. The average response rate per session was also computed for each rat. Analyses of variance (Perruchet, 1982) were performed on individual raw data (leverpresses per $10 \mathrm{sec}$ ) for each session by comparing the pretone and the tone level of pressing for the $\mathrm{CS}+$ and the $\mathrm{CS}-$. The standard suppression ratio $\mathrm{A}: \mathrm{A}+\mathrm{B}(\mathrm{A}=$ leverpressing during the tone; $\mathrm{B}=$ leverpressing before the tone) was also computed and used as a convenient index of conditioning for graphic representation.

\section{Off-Line Test Sessions}

Three off-line recording test sessions were conducted during this experiment. The first session (reference session) took place $24 \mathrm{~h}$ before the first habituation session, the second (Test 1) after four sessions of the initial discrimination, and the third (Test 2) after four sessions of reversal. These tests were conducted in the same experimental cage as was the conditioning, but the magazine and the lever of the instrumental task were removed. During each of these sessions, four frequencies were presented in a pseudorandom order: $1,4,10$, and $14 \mathrm{kHz}$. Each tone was of $1-\mathrm{sec}$ duration; the ITI was $1 \mathrm{~min}$ in average (range $=45$ to $75 \mathrm{sec}$ ). Thus, during these sessions, the animals were submitted both to the two frequencies used as $\mathrm{CS}+$ and $\mathrm{CS}$ - during conditioning and to two other frequencies close to the $\mathrm{CS}+$ and close to the CS - . The first stimulus presentation occurred about $15 \mathrm{~min}$ after the animal was placed in the cage and, for Tests 1 and 2, the CS + frequency was never used as a first stimulus (to avoid a "surprising" or arousal effect).

\section{Collection and Analysis of Cellular Data}

The recording procedure was the same as that described in an earlier study (Edeline et al., 1988). The multiunit activity (MUA), recorded through field effect transistors (input $15 \mathrm{pA}$, output $3 \mathrm{k} \Omega$ ) placed on the animal's head, was fed to a preamplifier $(300-10000 \mathrm{~Hz}$ ) and sent into a highpass filter (cut off at $400 \mathrm{~Hz}$ ). The output of this filter, displayed on an oscilloscope, was recorded on a magnetic tape during the $5 \mathrm{sec}$ of pretone and the $1 \mathrm{sec}$ of tone. During the off-line analysis, the MUA was fed into a voltage window discriminator to select the largest spikes (signal to noise $2: 1$ ). The output pulses were stored on each trial in successive 10 -msec bins during the $200 \mathrm{msec}$ of pretone and the first $400 \mathrm{msec}$ of tone. Standard computer programs allowed construction of individual histograms, which were then averaged to give group histograms. Ten (out of 12) channels of multiunit activity, showing the strongest auditory responses, were selected for each anatomical structure for group histograms. The same procedure as those previously described by Edeline et al. (1988) were used to avoid neural or nonneural feedback coming from the animal movements. We discarded trials in which movements of large amplitude were observed; electrical artifacts were ruled out (on the basis of their magnitude and rise time) by a two-threshold trigger (Courtice, 1975). Finally, the pretone firing rate was submitted to an analysis of variance across sessions in order to detect gradual shifts of firing rate across days.

To compare the sensory response to tones (three or four bins after tone onset) across different recording sessions, normalized $z$ scores were computed at each trial by subtracting the mean firing rate in the 20 pretone bins from the rate in the first three or four bins following tone onset, then the difference was divided by the standard deviation of the pretone activity. These normalized data took into account possible differences of pretone firing rate from one trial to another or from one electrode placement to another. All statisti$\mathrm{cal}$ analyses were performed by analyses of variance on the $z$ scores computed at each trial. For each structure, the analysis of variance involved the recorded electrodes as subject factors $(n=10)$ and orthogonal repeated measures as factors of sessions (three levels: reference, Test 1 , and Test 2 ), stimulus [four levels: the CS + , the $\mathrm{CS}(+)$, the $\mathrm{CS}(-)$, and the $\mathrm{CS}-1$, and time bin (four levels: four 10 -msec intervals after stimulus onset). Intersession comparisons (between the reference session and the 2 test sessions), as well as intrasession comparisons (between the different frequencies), were made to detect both the increases of responsiveness due to conditioning and the selectivity of these increases of responses. Again, because the goal was to observe the modifications of preexisting sensory responses, the analyses were restricted (after examination of the histograms) to the time bins exhibiting "on" responses. Thus, we analyzed the first two bins (0-20 msec) after tone onset for the DCN, the second through the fifth bin $(10-50 \mathrm{msec})$ for the MG, and the first four bins $(0-40 \mathrm{msec})$ for the $\mathrm{AC}$.

\section{Histology}

At the end of the experiment, the animals were perfused (intracardiac perfusion) under deep pentobarbital anesthesia with $0.9 \%$ saline followed by $10 \%$ formaline. The brains were kept 2 weeks in $10 \%$ formaline, frozen, sliced at $60 \mu \mathrm{m}$, and stained with cresyl violet for Nissl preparation.

\section{RESULTS}

\section{Behavioral Results}

\section{Habituation}

As shown in Figure 1, during the habituation, no significant decrease of leverpressing for food was observed during the presentation of either the $1-\mathrm{kHz}$ tones or the 14-kHz tones. No difference can be detected between the pretone and the tone leverpressing for the two frequencies at the first habituation session $[F(1,5)<1$, for both tones] or at the second habituation session $[F(1,5)=2.33$, n.s., and $F(1,5)<1]$.

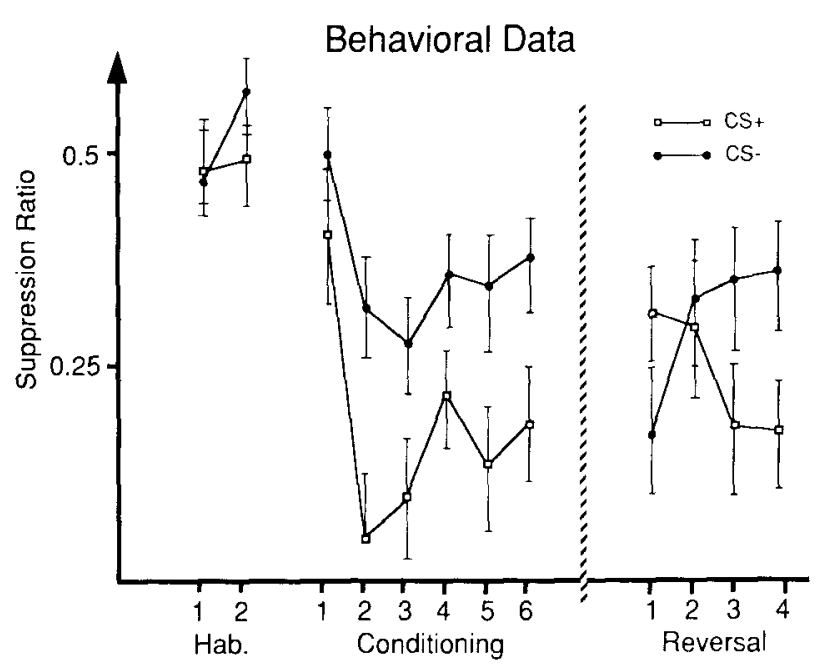

Figure 1. Averaged ( $n=6$ ) suppression ratio $\mathrm{A}: \mathrm{A}+\mathrm{B}(\mathrm{A}=$ leverpressing responses during the $10 \mathrm{sec}$ of tone; $B=$ leverpressing responses during $10 \mathrm{sec}$ before tone) for the $\mathrm{CS}+$ and the $\mathrm{CS}$ - during conditioning and its reversal. Note that, in habituation, no suppression was observed to the $1000-\mathrm{Hz}(\bullet)$ or the $14000-\mathrm{Hz}$ ( $\square$ ) tone presentations and that a clear discriminative effect was observed after two conditioning sessions. Note also the clear reversal of the behavioral responses after four sessions of reversal. 


\section{Initial Discrimination}

At the first conditioning session, no statistical decrease of leverpressing was observed for the CS $+[F(1,5)=3.62$, n.s.] or the CS $-[F(1,5)<1]$. However, a significant difference $[F(1,5)=17.66, p<.01]$ existed between the rates of leverpressing during the $\mathrm{CS}+$ presentations and during the $\mathrm{CS}-$ presentations, which is corroborated by the interaction $[F(1,5)=32.32, p<.005]$ between the factor of period (before and during stimulus presentation) and the factor of type of trial (CS + vs. CS - ). On the second day of training, a strong suppression was observed during the CS + presentations $[F(1,5)=65.67, p<.001]$. A slight suppression was also observed during the CS presentations $[F(1,5)=11.01, p<.05]$, but the interaction between the period and type of trial factors was strongly significant $[F(1,5)=55.27, p<.001]$; thus, the suppression to the CS + was greater than that to the CS - . During the subsequent sessions, the suppression of leverpressing remained significant for the CS + until the sixth session, whereas the suppression was significant for the CS - at the fourth session. The interaction between period and type of trial remained significant from the third session $[F(1,5)=55.08, p<.001]$ to the sixth session $[F(1,5)=71.13, p<.001]$. This indicates that the suppression to the CS + was statistically different from that to the CS - at all the sessions of the initial discrimination.

\section{Reversal}

During the first reversal discrimination session, the behavioral responses clearly corresponded to the previously learned discrimination. The suppression of leverpressing was stronger to the previous CS + than to the new CS + $[F(1,5)=27.02, p<.05]$. During the next two sessions, a progressive reversal of the behavioral responses was observed. At the third session, the suppression was greater for the new $\mathrm{CS}+$ and the interaction between period and type of trial was significant $[F(1,5)=32.19$, $p<.01]$, even when the animal still showed a suppres-
REF.
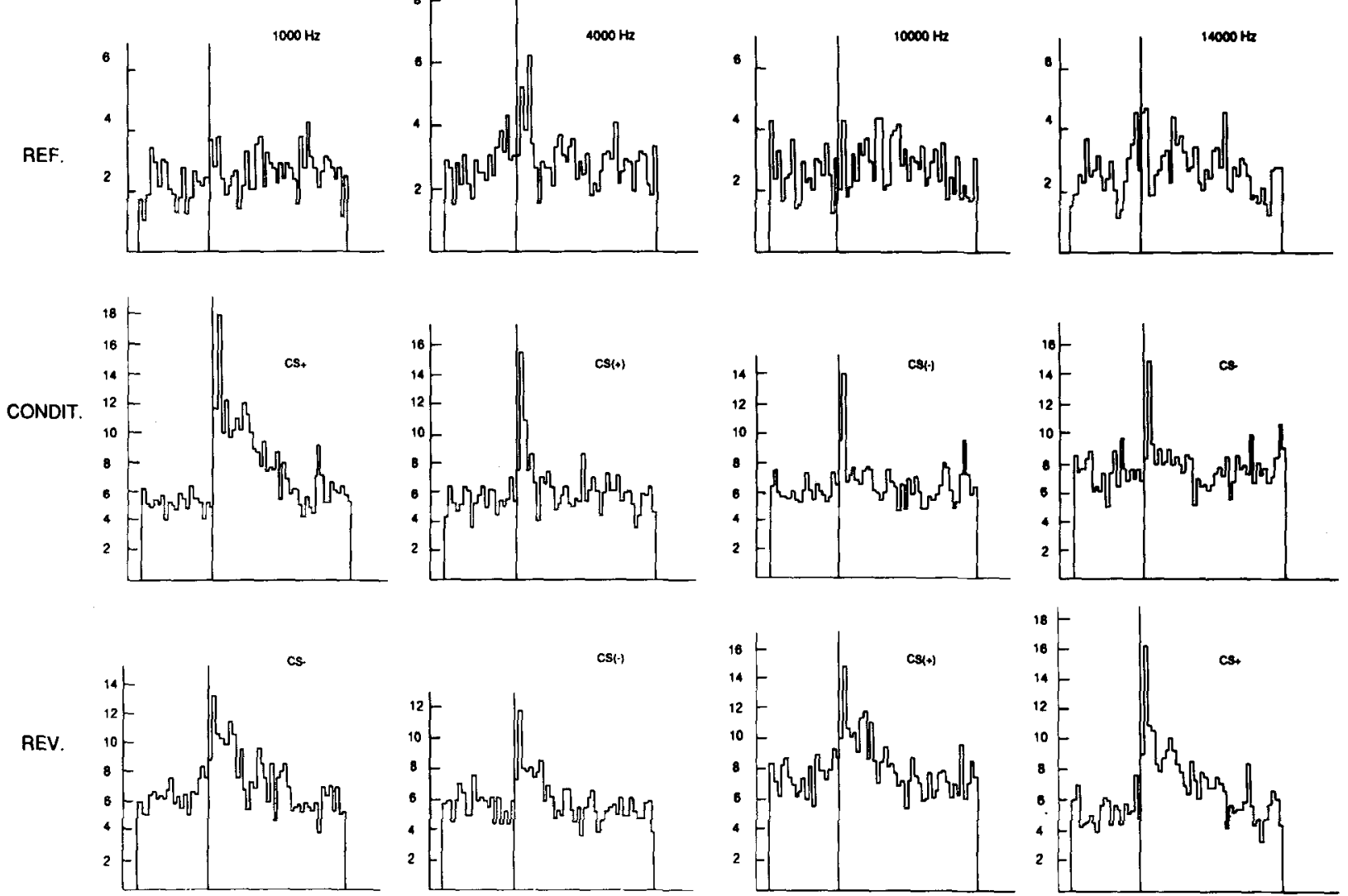

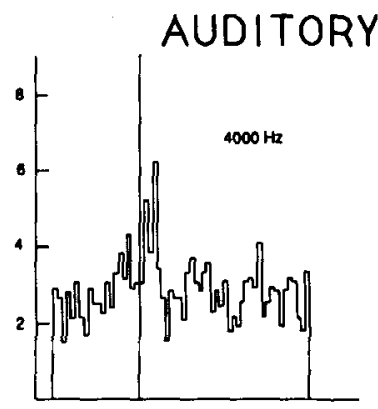

Figure 2. Averaged group histograms ( $n=10$, bin width $=10 \mathrm{msec}$ ) derived from the cortical placements before conditioning (REF), after $\mathbf{4 0}$ trials of conditioning (CONDIT), and after 40 trials of reversal (REV). For the reference test session, the four different frequencies $(1,4,10$, and $14 \mathrm{kHz})$ are labeled for the reference test (before conditioning). For the tests conducted during the discrimination and its reversal, the tones are named $\mathrm{CS}+, \mathrm{CS}(+)$ (for the closest frequency of the $\mathrm{CS}+$ ), $\mathrm{CS}-$, and $\mathrm{CS}(-)$ (for the closest frequency of the $\mathrm{CS}-$ ). Note that, after $\mathbf{4 0}$ trials of conditioning, the CS + elicited the strongest response, even compared with the closest frequency of the CS +. Note also that after $\mathbf{4 0}$ trials of reversal, the strongest responses were obtained for the new CS + presentations, even compared with the $\mathrm{CS}(+)$. 
sion to the previous CS $+[F(1,5)=10.54, p<.05]$. The reversal was achieved at the fourth session, in which no suppression of leverpressing to the new $\mathrm{CS}$ - was observed $[F(1,5)=4.2$, n.s.]; however, there was suppression to the new CS + presentation $[F(1,5)=8.33$, $p<.05]$.

\section{Electrophysiological Results}

\section{Auditory Cortex}

Reference test session. As shown in the histograms in Figure 2, only slight "on" responses were observed to tone presentations before conditioning, with no difference of response magnitude between frequencies $[F(1,9)<1$, in all cases]. Possible reasons for these weak responses will be provided in the Discussion section.

Test 1. After 40 trials of the initial discrimination, marked increases in responsiveness were observed for the four frequencies tested (Figure 2, middle row). These increases were noted on the first four bins after tone onset, all of which had significantly greater responses than dur- ing the reference test session [for the first four bins, respectively: $F(1,9)=35.17, p<.001 ; F(1,9)=43.81$, $p<.001 ; F(1,9)=67.13, p<.001 ; F(1,9)=31.29$, $p<.001]$. The mean $z$ scores, plotted for each frequency in Figure 3, show that the strongest increases occurred for the frequencies used as CS + during conditioning, except on the third bin of tone in which the responses to the $\mathrm{CS}+$ and to the closest frequency increased similarly. A statistical difference exists between the $z$ scores obtained for the CS + and the frequency closest to the CS + on the first $[F(1,9)=11.18, p<.05]$ and the second bin of tone $[F(1,9)=20.88, p<.001]$. On the third and the fourth bin of tone, the responses to the CS + and the closest frequency were not statistically different [respectively, $F(1,9)<1$, and $F(1,9)=3.82$, n.s.].

Test 2. After four sessions of reversal (Figure 2, bottom histograms), the strongest responses were now observed for the new CS + . As shown in Figure 3, the responses at all the frequencies remained clearly greater than those during the reference test session. The selectivity observed at Test Session 1 had disappeared and, on

\section{AUDITORY CORTEX}

(Z-scores)
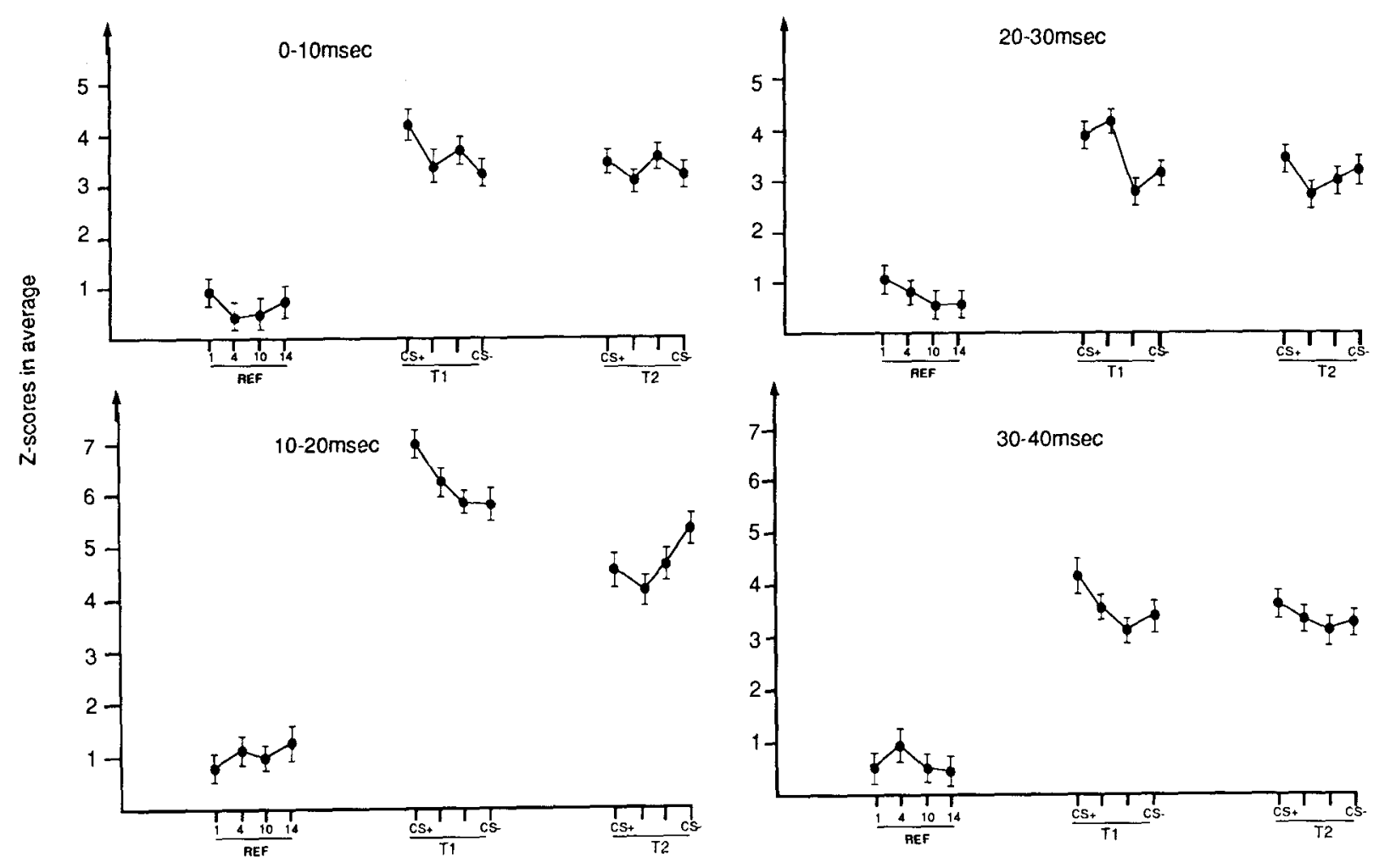

Figure 3. Averaged $z$ scores $(n=10 \pm S E$ ) for the first, second, third, and fourth bins of tone period before conditioning (REF), after 40 trials of conditioning (Test 1 [T1]), and after 40 trials of reversal (Test 2 [T2]). Note the clear generalization gradients obtained for all the temporal windows during Test 1 , in which the $C S+$ elicited the strongest responses. Note also that, during Test 2 , after 40 reversal trials, these gradients had disappeared and the greatest $z$ scores then were for the new CS + on the second bin of tone (10-20 msec). 
the second bin after tone onset, the $z$ scores corresponding to the new CS + were significantly greater than those to the closest frequency $[F(1,9)=10.28, p<.05]$.

\section{Medial Geniculate Data}

Reference test session. During the reference test session (Figure 4, top panel), the same responses were obtained to the four frequencies. As shown in Figure 5, no difference of responsiveness was detected by comparing the mean $z$ scores obtained for each frequency $[F(1,9)<1$, in all cases] in this session.

Test 1. During this test session, an increase of the "on" response was observed for the frequency used as $\mathrm{CS}+$ during conditioning (Figure 4, middle panel). This increase of response occurred mostly on the third and fourth bin after tone onset (20-40 msec after tone onset). At these time bins (see Figure 5), the responses for the frequencies used as $\mathrm{CS}+$ were significantly increased, relative to those in the reference test session $[F(1,9)=13.11$, $p<.05$, and $F(1,9)=16.34, p<.05$, respectively, for the third and the fourth bins of tone]. Moreover, at these bins, the $z$ scores for the CS + were different from those obtained for the closest frequency (i.e., either 4 or $10 \mathrm{kHz})[F(1,9)=9.07, p<.05$, and $F(1,9)=10.11$, $p<.05$, for the third and fourth bins of tone]. There was no difference between the responses to the CS+ and those to the frequency close to the CS $+[F(1,9)=1.28$, n.s. $]$ on the second bin of tone.

Test 2. During Test 2 (Figure 4, bottom panel), the greatest increases of response occurred for the new CS + . The mean $z$ scores obtained in this session (see Figure 5) showed that the selectivity observed in Test 1 had shifted to the new CS + on the third and fourth bin of tone. There was an increase in responses for the frequency used as $\mathrm{CS}+$, relative to those in the reference test session $[F(1,9)=12.56, p<.05]$. The $z$ scores of the new $\mathrm{CS}+$ were statistically different $[F(1,9)=8.87, p<.05]$ from the $z$ scores of the new CS - (previous CS+). Moreover,

\section{MEDIAL GENICULATE}
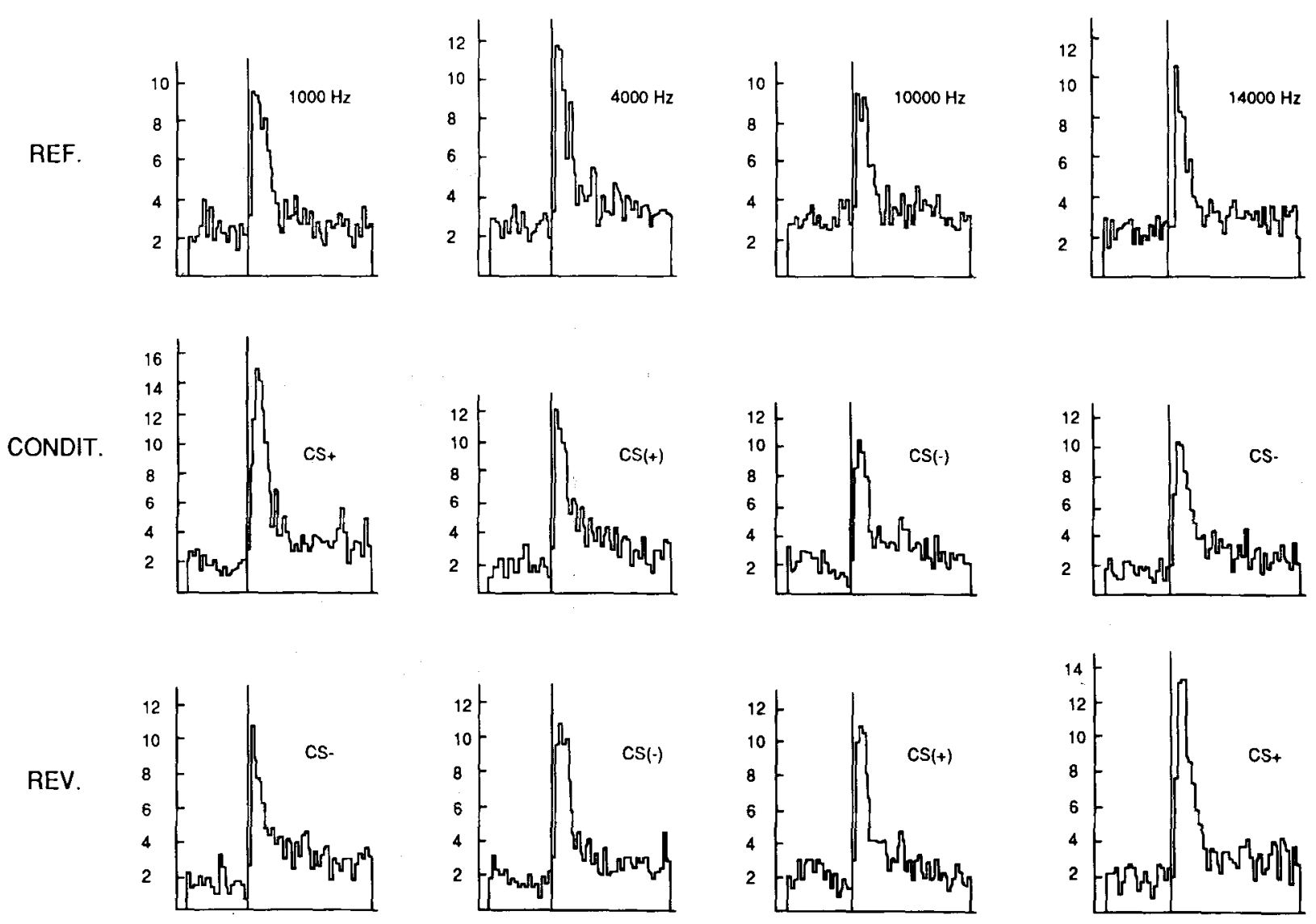

Figure 4. Averaged group histograms ( $n=10$, bin width $=10 \mathrm{msec}$ ) recorded in the $\mathrm{mMG}$ during the reference session before conditioning (REF), after $\mathbf{4 0}$ trials of the initial discrimination (CONDIT), and after $\mathbf{4 0}$ trials of reversal (REV). Note that similar responses were observed to the four frequencies before conditioning and that after $\mathbf{4 0}$ conditioning trials the greatest responses were obtained for the $\mathrm{CS}+$, even compared with the $\mathrm{CS}(+)$. Note also that, after 40 trials of reversal, the greatest responses were detected during the new CS + presentations. 
a significant discriminative effect between $\mathrm{CS}+$ and its closest frequency was detected in the average of the third and fourth bins after tone onset $[F(1,9)=7.14, p<.05]$.

\section{Dorsal Cochlear Nucleus}

Reference test session. Before conditioning (Figure 6, top panels), the different frequencies elicited consistent responses and no statistical difference of responsiveness between frequencies was detected by comparing the mean $z$ scores (plotted in Figure 7) for the first and second bins of tone $[F(1,9)<1$, in all cases $]$.

Test 1. As shown in Figures 6 and 7, an increase of the "on" response elicited by the CS + during the first $10 \mathrm{msec}$ after tone onset was observed after 40 trials of the initial discrimination. However, the response to the frequency close to the $\mathrm{CS}+$ frequency showed the same degree of enhancement. On the first bin of tone, there was a significant difference between the responses to the $\mathrm{CS}+$ and those to the $\mathrm{CS}-[F(1,9)=23.46, p<.001]$, but no significant difference existed between the responses to the CS + and those to the closest frequency to the CS + $[F(1,9)=1.19$, n.s. $]$. No change in response was detected on the second bin after tone onset compared with the reference test session $[F(1,9)<1$, in all cases], and no difference between frequencies was noted by comparing the $z$ scores related to each frequency.

Test 2. As shown in Figures 6 and 7, the increases of response previously detected at Test 1 were no longer present, and no change corresponding to the new reinforcement rule was observed on this session. No significant difference was noted on the first bin of tone between the different frequencies $[F(1,9)<1$, in all cases]; no difference was detected between these responses and those elicited during the reference test session. The analyses per-

\section{Medial Geniculate (Z-Scores)}

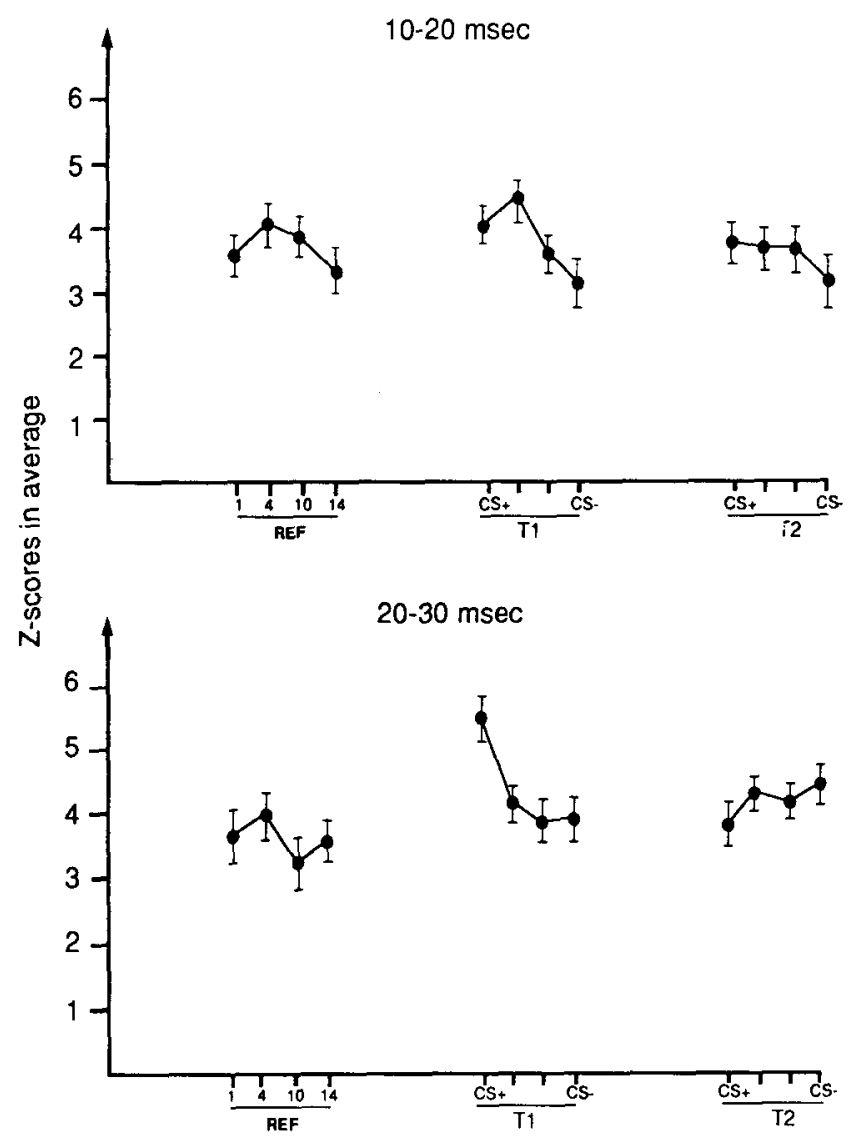

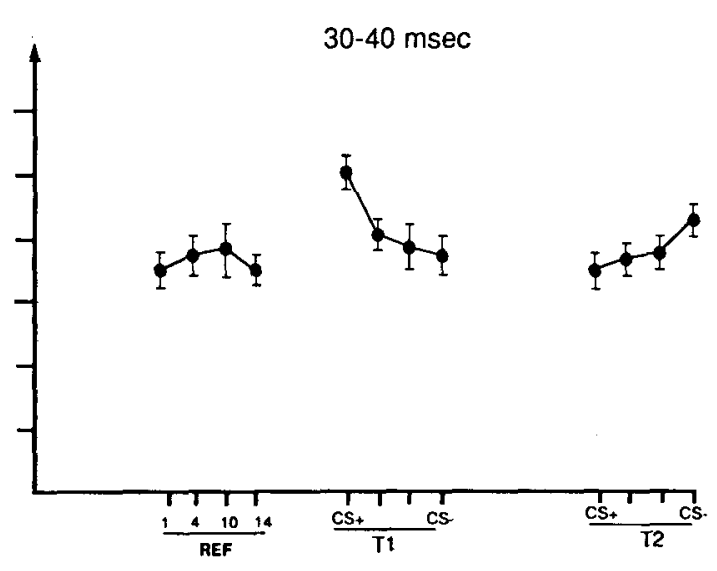

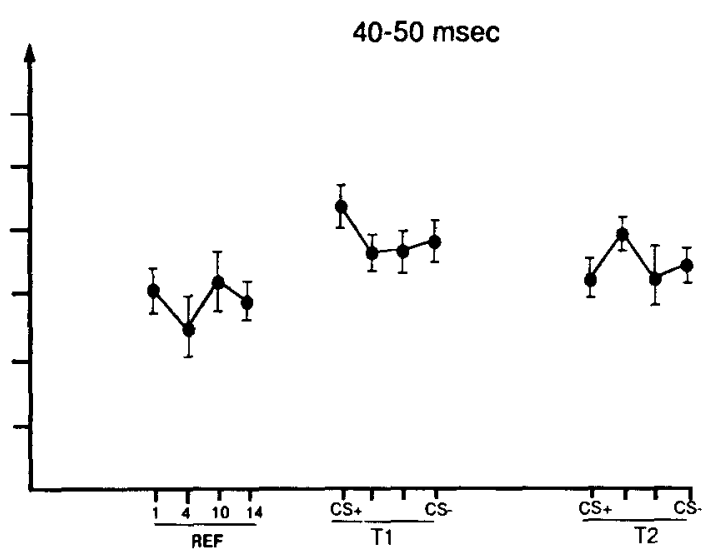

Figure 5. Averaged $z$ scores $(n=10 \pm S E$ ) derived from $\mathrm{mMG}$ recording for the second, third, fourth, and fifth 10 -msec bins of tone period, during the reference session (REF), the test sessions after 40 conditioning trials (Test 1 [T1]), and the test sessions after 40 trials of reversal (Test 2 [T2]). Note that similar responses were observed before conditioning (in REF) regardless of the temporal window and that, after 40 conditioning trials, the responsiveness for the CS + frequencies was clearly greatest especially on the third and fourth bins of tone. Note also that, during Test 2 , after 40 trials of reversal, the generalization gradient observed in Test 1 had disappeared and the new $\mathrm{CS}+$ elicited the strongest responses in the same temporal window (20-30 and $30-40$ msec after tone onset). 
formed on the second, third, and fourth bins after tone onset did not reveal changes in responses at this stage of the experiment.

\section{Electrodes Placements (see Figure 8)}

At the thalamic level, correct electrode placements were found in the mMG for six recording loci and in the suprageniculate immediately above the mMG for four other recording loci. No difference in tone responses were observed between these two locations. All of the cortical recordings were from area TE1 of the Zilles (1985) atlas. This region corresponds, at least partially, to the cortical projection area of the MG in the rat (see Ledoux, Ruggiero, \& Reis, 1985; Roger \& Arnault, 1989). Recordings in the cochlear nucleus were from the dorsal subdivision of this nucleus, mainly (8/10 placements) in the fusiform cells layer; two recording loci were located in the molecular layer of this subdivision.

\section{DISCUSSION}

The major results of this study can be summarized as follows: Pairing one tonal frequency with an electrical footshock (as the US) during discrimination conditioning induced specific modification of responses at the level of the auditory cortex (AC) and the magnocellular medial geniculate (mMG) and less specific changes in the dorsal cochlear nucleus (DCN). The reversal induced new modifications at the levels of the AC and $\mathrm{mMG}$, but not at the level of the DCN. Both during the initial and reversed discrimination, the difference of leverpressing for food responses during $\mathrm{CS}+$ and $\mathrm{CS}-$ presentations indicated that these neural changes were correlated with the acquisition of a discrimination between frequencies.

\section{Methodological Comments}

Recording multiunit activity in free-moving animals always raises some methodological problems, because it is impossible to control the sensory input constancy. We have previously discussed these problems (Edeline et al., 1988), and we emphasize here that the action of the middle ear muscle has a latency of 35-70 msec (Carmel \& Starr, 1963) in the cat at rest and can only account for decreases of evoked responses (Start \& Livingston, 1963). Moreover, as a random presentation of the different frequencies was given during the different test sessions, it

\section{COCHLEAR NUCLEUS}
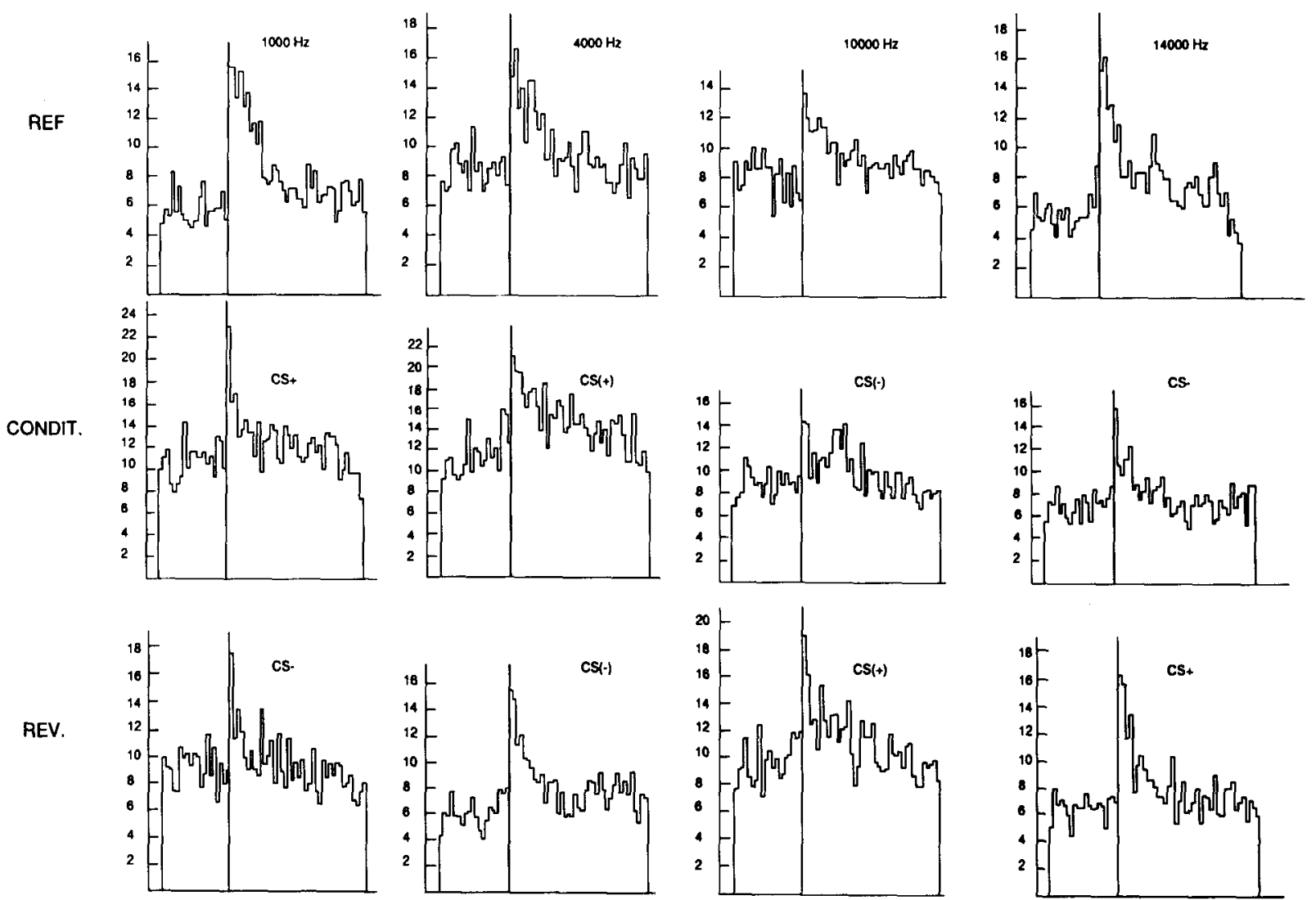

Figure 6. Averaged group histograms $(n=10$, bin width $=10 \mathrm{msec})$ derived from the dorsal cochlear nucleus before conditioning (REF), after 40 trials of conditioning (CONDIT), and after 40 trials of reversal (REV). Note that similar responses to the four frequencies were observed before conditioning and that, after 40 conditioning trials, the CS + and the $\operatorname{CS}(+)$ elicited the strongest responses, compared with the $\mathrm{CS}-$ and the $\mathrm{CS}(-)$. Note also that, during Test 2, after 40 reversal trials, the changes observed in Test 1 were dissipated, but the new CS + and its closest frequency did not elicit stronger responses than did the new CS- and its closest frequency. 


\section{COCHLEAR NUCLEUS}

(Z-Scores)
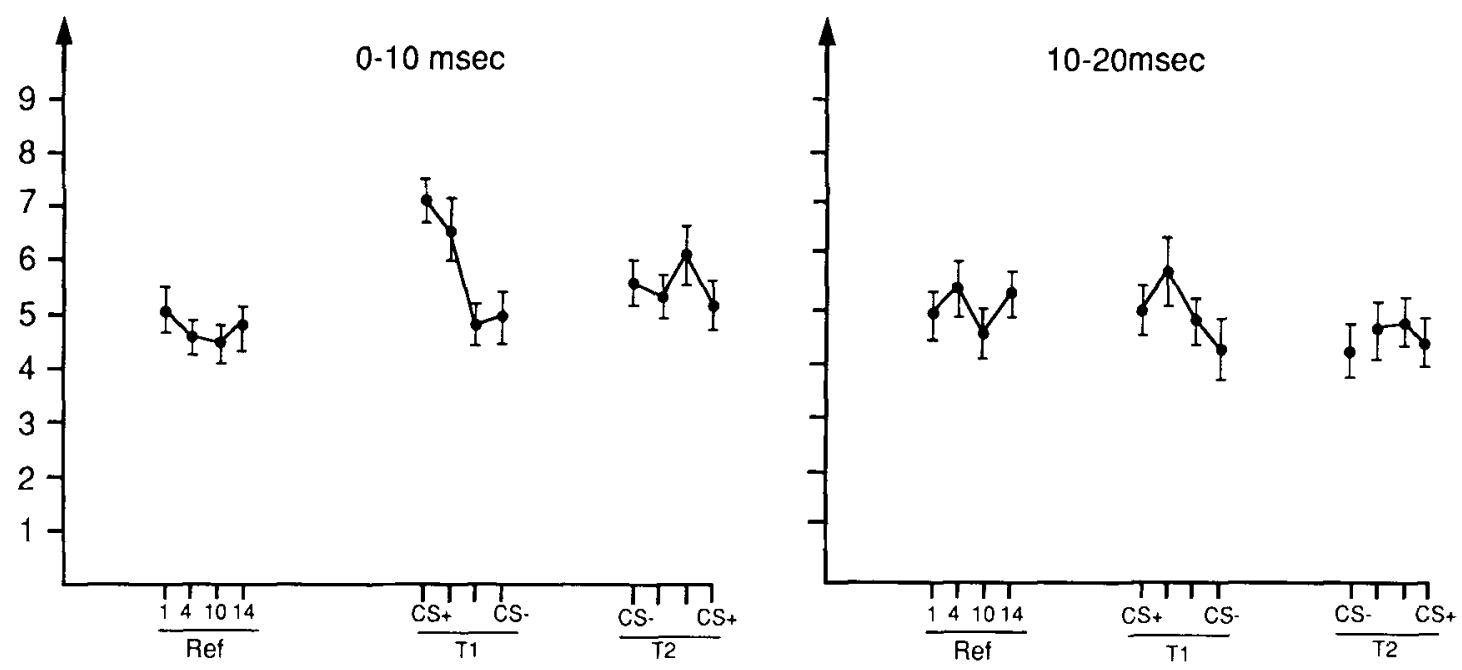

Figure 7. Averaged $z$ scores $(n=10 \pm S E$ ) derived from the dorsal cochlear recording before conditioning (REF), after 40 conditioning trials (Test 1 [T1]), and after 40 trials of reversal (Test 2 [T2]) for the first and second bins of tone period. Note the similar responsiveness to the four frequencies before conditioning and the strong responses to the $\mathrm{CS}+$ and the $\mathrm{CS}(-)$ in Test 1 on the first bin of tone. Note also that no difference of responsiveness was observed after 40 trials of reversal (Test 2) on the first or the second bin of tone.

is unlikely that the animals could adopt a specific orientation or location with regard to the speaker before the occurrence of one of the frequencies. Finally, it is quite difficult to attribute the present results to a startle response at the CS + presentations, since it is unlikely that this response (6-16 msec in latency; for a review, see Davis, 1984) can affect the neural activity of each structure on different time bins after tone onset.

\section{Comparisons With Physiological Data}

Before discussing the present results in terms of associative learning, four points must be addressed regarding the auditory physiology.

First, the fact that the different frequencies used in our study elicited similar responses before conditioning can be attributed to (1) multiunit recordings with low impedance electrodes, (2) the intensity used $(75 \mathrm{~dB})$, which was far from the threshold, thus making the frequency selectivity less obvious, and (3) the average of different electrode placements. Because none of the recording sites showed a strong selectivity before conditioning, we believe that the first two points are the most important. The fact that the two subcortical structures belong to the nonlemniscal system (see Graybiel, 1972, for definition), in which many cells are broadly tuned, certainly contributes to this lack of selectivity.

Second, the averaged response pattern emerging from our cochlear nucleus recordings is an "on" response rather than a sustained response that can be explained by the properties of the DCN cells. It is well known that sus- tained responses are rare, relative to those found in the ventral division of the nucleus. Also, Evans and Nelson (1973) have stressed the fact that, with unanesthetized preparations, there is a dramatic increase of the number of units showing inhibition response ( $92 \%$ of the units), compared with anesthetized preparations $(8 \%)$. The work of Young and Brownell (1976) and, more recently, of Rhode and Kettner (1987) have confirmed that a great proportion of units shows an "onset inhibition" pattern in unanesthetized animals.

Third, at the level of the mMG, broad, as well as sharp, tuning curves have been described in the anesthetized cat (Aitkin, 1973; Calford, 1983; Morel, Rouiller, DeRibeaupierre, \& DeRibeaupierre, 1987), and the lack of tonotopy of this portion of the MG has been reported in many studies. Thus, it is not very surprising that pooling data from multiunit recordings yielded similar responses to the four frequencies.

Finally, the lack of responsiveness before learning in the AC contrasts with the strong responses observed after the beginning of conditioning, as well as during electrode implantations. We have observed this phenomenon previously (Edeline, Neuenschwander-El Massioui, \& Dutrieux, 1990), and, since strong responses were not observed in other studies performed under similar conditions (Disterhoft \& Stuart, 1976; Olds et al., 1972), we have attributed this to the polymodality of the temporal cortical area in the rat. The absence of marked degeneration in any layer of the rat temporal cortex after MG lesions (Vaughan, 1980) supports such a hypothesis. Moreover, the same type of observation has recently been 

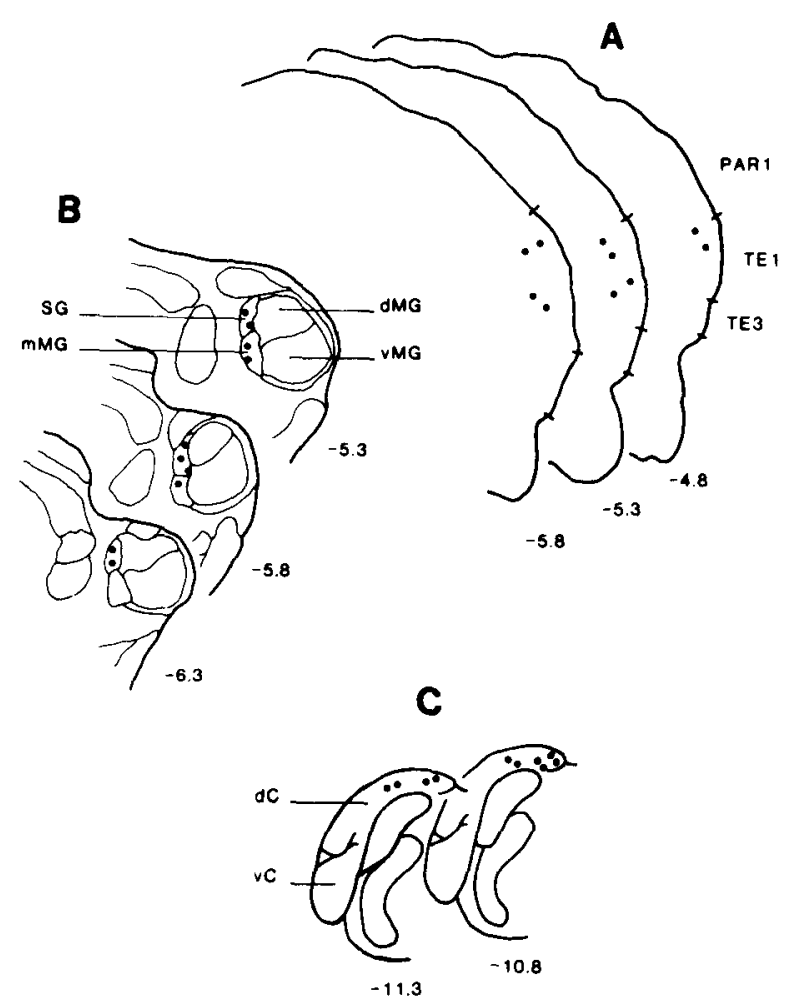

Figure 8. Outlines showing the placements of the recording electrodes in the auditory cortex (A), the medial geniculate $(B)$, and the dorsal cochlear nucleus (C). The boundaries between the cortical areas are from Roger and Arnault (1989), the arrangement of the MG subdivisions are from the study of Ledoux et al. (1985), and the dorsal cochlear nucleus limits are from Mugnaini, Warr, and Osen (1980).

reported (Diamond \& Weinberger, 1989) at the singleunit level: Neurons weakly responsive to tones before conditioning exhibited significant evoked activity to numerous frequencies after conditioning. As proposed by these authors, it is quite possible that learning induces the expression of subthreshold input.

\section{Relationship to Previous Studies}

The work of Buchwald et al. (1966) was the first to show that conditioned changes can be detected at many levels of the CS pathway, even at the cochlear nucleus. Oleson et al. (1975) have clearly demonstrated the associative nature of the changes that occur at the cochlear nucleus, finding a differential effect to a CS + compared to a CS-; however, they could not address the question of the frequency specificity (because they used a discrimination between a tone and a white noise). Our present results suggest that the conditioned changes at the level of the DCN can be frequency specific, but they cannot show the degree of selectivity observed in the auditory forebrain. Two possibilities can explain the occurrence of changes at the lower level of the CS pathway: (1) these changes can reflect efferent controls upon the cochlear nucleus, or (2) they can reflect intrinsic changes at this level. Oleson et al. (1975) have claimed that the changes they have observed at the cochlear level were probably the result of a "descending excitation coming from the AC" (p. 1136). However, two of their placements in the posteroventral nucleus reached the learning criteria in five trials, far more rapidly than in the cortex. It was not our goal in the present study to determine if changes at the cochlear nucleus level are intrinsic or extrinsic. We can only point out that there is no reason for a centrifugal influence (regardless of the structure it comes from) to act after $\mathbf{4 0}$ trials of conditioning and not after 40 trials of reversal and that it seems unlikely that a centrifugal influence would affect only the first bin after tone onset for the CS + and its closest frequency, as was the case during Test 1.

In contrast with the changes occurring at the DCN level, which were only increases in responses to the low or high frequencies, the changes in the auditory forebrain seem to be able to shape a gradient of response around a specific frequency and to reorganize this gradient when the significance of the frequency changes. At the cortical level, the observation of frequency-specific changes is not surprising, since the work of Diamond and Weinberger $(1986,1989)$ has firmly established that shifts of tuning curves occur after classical conditioning in the auditory cortex. Our results raise the possibility that "cluster" recordings can allow detection of frequency-specific changes. However, the changes observed here (as in all multiunit studies) were only increases of responses, whereas both increases and decreases of evoked activity can be detected at the single-unit level (Diamond \& Weinberger, 1984; Weinberger, 1982; Weinberger, Hopkins, $\&$ Diamond, 1984). This can perhaps be explained by the fact that the construction of a "multiple units" histogram by summing the data of 21 single-unit recordings in the auditory cortex revealed an increase of evoked response, relative to sensitization (Weinberger et al., 1984), despite the fact that some of these units showed decreases during conditioning.

At the thalamic level, the study of Gabriel, Miller, and Saltwick (1976) showed that discriminative effects and their reversals can be observed in the mMG. The major difference between their study and the present one is the off-line collection of the neural data in the present study, which allowed testing of the selectivity of the learninginduced modification in absence of the conditioned fear induced by the tone-shock pairing. In addition, this type of procedure also helps prevent subtle nonassociative processes, which can possibly occur during the acquisition of a discrimination (Birt, Nienhuis, \& Olds, 1979), biasing the short-latency sensory changes. In fact, receptive fields modifications observed in the auditory cortex after classical conditioning have indicated that learninginduced plasticity can be expressed in a different situation (or "context") from that present for the learning (Diamond \& Weinberger, 1989). The present data suggest that this can also be the case in some subcortical structures, because the changes reported here occurred in a different situation from the learning situation (absence of 
US, presence of other frequencies, tones of shorter duration, and absence of the bar and the food magazine used for the on-line behavioral responses).

\section{Relationships Between Behavioral and Neural Changes}

At the behavioral level, the slight decreases of leverpressing for food to the CS - can be explained by the fact that, during conditioning, the animals always exhibited much more orienting reactions to acoustic stimuli than they did during habituation. We suspect that all of the unconditioned reactions (and especially the orienting responses) led to decreases in leverpressing for food to CS - presentations. The increase in attention to all acoustic stimuli during conditioning may be reflected in the changes observed at the cortical level: Marked increases of responses appeared for the four frequencies, even though the largest increases were to the CS + . At the single-unit level, either frequency-specific changes or general changes were reported after classical conditioning (Diamond \& Weinberger, 1986, 1989). It is quite possible that both general increase and specific increases at the $\mathrm{CS}+$ frequency can be observed in multiunit recordings. In our experiment, the subcortical sensory responses seemed less influenced by the general increase of attention to auditory stimuli. This may suggest that the effects observed at the cortical level reflect more than one unique phenomenon, whereas the changes observed in the subcortical structures reflect only the pairing effects. On the other hand, the fact that the specific changes observed in the $\mathrm{AC}$ occurred with shorter latencies than those in the thalamus suggests that the specific thalamic changes possibly are "shaped" by a centrifugal cortical influence (as proposed by Gabriel, 1976). This explanation is more unlikely at the level of the DCN since (1) there are no direct connections between the AC and the DCN and (2) the changes in the DCN occurred at short latency.

Taken together, the present data indicate that, during acquisition of a discrimination between frequencies, learning can induce the formation of an "adaptive filter" at several levels of the CS sensory pathway. This adaptive filter is able to shift relatively rapidly and selectively at the level of the auditory forebrain, whereas it seems broader at the more peripheral levels and less easily "reshaped" when the significance of the frequencies changes across time. Quantitative investigations (frequency-receptive field analyses) must now be conducted to determine the precise learning effects on the filtering ability of single neurons at each level of the nonlemniscal auditory pathway.

\section{REFERENCES}

AItKIN, L. M. (1973). Medial geniculate body of the cat: Responses to tonal stimuli of neurons in medial division. Journal of Neurophysiology, 36, 275-283.

Bakin, J., Condon, C. D., Weinberger, N. M. (1988). Learning specifically alters frequency receptive fields in the auditory cortex of guinea pigs. Society for Neuroscience Abstracts, 14, 862 (Abstract No. 344.13).
BIRT, D., \& OLds, M. (1981). Associative response changes in lateral midbrain tegmentum and medial geniculate during differential appetitive conditioning. Journal of Neurophysiology, 46, 1039-1055.

BIRT, D., NiEnhuIS, R., \& OLDS, M. (1979). Separation of associative from non-associative short latency changes in medial geniculate and inferior colliculus during differential conditioning and reversal in rats. Brain Research, 167, 129-138.

Buchwald, J. S., Halas, E. S., \& Schramm, S. (1966). Changes in cortical and subcortical unit activity during behavioral conditioning. Physiology \& Behavior, 1, 11-22.

CALFORD, M. B. (1983). The parcellation of the medial geniculate body of the cat defined by the auditory responses properties of single units. Journal of Neuroscience, 3, 2350-2365.

Carmel, P. W., \& Starr, A. (1963). Acoustic and non-acoustic factors modifying middle-ear muscles activity in awake cats. Journal of Neurophysiology, 26, 598-616.

Courtice, C. J. (1975). Action potential selection by amplitude and width. Journal of Physiology (London), 246, 18-P.

DAvis, M. (1984). The mammalian startle response. In R. C. Eaton (Ed.), Neural mechanisms of startle behavior (pp. 287-351). New York: Plenum.

Diamond, D. M., \& Weinberger, N. M. (1984). Physiological plasticity of single neurons in auditory cortex of the cat during acquisition of the pupillary conditioned response: Secondary field (AII). Behavioral Neuroscience, 98, 198-210.

Diamond, D. M., \& Weinberger, N. M. (1986). Classical conditioning rapidly induced changes in frequency receptive fields of single neurons in secondary and ventral ectosylvian auditory cortical fields. Brain Research, 372, 357-360.

Diamond, D. M., \& Weinberger, N. M. (1989). Role of context in the expression of learning-induced plasticity of single neurons in auditory cortex. Behavioral Neuroscience, 103, 471-494.

DisterhofT, J. F., Stuart, D. K. (1976). Trial sequence of changed unit activity in the auditory system of alert rat during conditioned response acquisition and extinction. Journal of Neurophysiology, 39, 266-281.

Disterhoft, J. F., \& Stuart, D. K. (1977). Different short latency response increases after conditioning in inferior colliculus of alert rat. Brain Research, 130, 315-334.

Edeline, J.-M., Dutrieux, G., \& Neuenschwander-El Massioui, N. (1988). Multiunit changes in hippocampus and medial geniculate body in free-behaving rats during acquisition and retention of a conditioned response to a tone. Behavioral \& Neural Biology, 50, 61-79.

Edeune, J.-M., Neuenschwander-El Massioni, N., \& DutrieuX, G. (1990). Discriminative long-term retention of multiunit changes in the hippocampus, the medial geniculate and the auditory cortex. Behavioral Brain Research, 39, 145-155.

Evans, E. F., Nelson, P. G. (1973). The responses of single neurones in the cochlear nucleus of the cat as a function of their location and anaesthetic state. Experimental Brain Research, 17, 402-427.

GABRIEL, M. (1976). Short-latency discriminative unit responses: Engram or bias? Physiological Psychology, 4, 275-280.

Gabriel, M., Miller, J. D., \& Saltwick, S. E. (1976). Multipleunit activity of the rabbit medial geniculate nucleus in conditioning, extinction, and reversal. Physiological Psychology, 4, 124-134.

Gabriel, M., Saltwick, S. E., \& Miller, J. D. (1975). Conditioning and reversal of short-latency multiunit responses in the rabbit medial geniculate nucleus. Science, 189, 1108-1109.

GRAYBIEL, A. M. (1972). Some fiber pathways related to the posterior thalamic region in the cat. Brain Behavior \& Evolution, 6, 363-393.

Halas, E. S., Bearlsey, J. V., \& Sandle, M. E. (1970). Conditioned neuronal responses at various levels in conditioning paradigms. Electroencephalography \& Clinical Neurophysiology, 28, 468-477.

Ledoux, J. E., Ruggiero, D. A., \& Reis, D. J. (1985). Projection to subcortical forebrain from anatomically defined regions of the medial geniculate body. Journal of Comparative Neurology, 242, 182-213.

Morel, A., Rouiller, E., DeRibeaupierre, Y., \& DeRibeaupierre, F. (1987). Tonotopic organization in the Medial Geniculate Body (MGB) of lightly anesthetized cats. Experimental Brain Research, 69, 24-42.

Mugnaini, E., WarR, W. B., \& OsEN, K. K. (1980). Distribution and light microscopic features of granule cells in the cochlear nuclei of cat, rat, and mouse. Journal of Comparative Neurology, 191, 581-606. 
Olds, J., Disterhoft, J. T., Segal, M., Kornalith, C. L., \& HiRsh, R. (1972). Learning centers of rat brain mapped by measuring the latencies of conditioned unit responses. Journal of Neurophysiology, 35, 202-219.

Oleson, T., Ashe, J., \& Weingerger, N. M. (1975). Modification of auditory and somatosensory activity during pupillary conditioning in the paralysed cat. Journal of Neurophysiology, 38, 1114-1139.

Paxinos, G., \& WATsON, C. (1982). The rat brain in stereotaxic atlas. New York: Academic Press.

Perruchet, P. (1982). Programme de description et d'analyses inférentielles de données expérimentales par microordinateur. Informatique \& Sciences Humaines, 85, 87-101.

RHODE, W. S., KetTNer, R. E. (1987). Physiological study of neurons in the dorsal and posteroventral cochlear nucleus of the unanesthetized cat. Journal of Neurophysiology, 57, 414-442.

Roger, M., ARnault, P. (1989). Anatomical study of the connections of the primary auditory area in the rat. Journal of Comparative Neurology, 287, 339-356.

Ryugo, D. K., Weinberger, N. M. (1978). Differential plasticity of morphologically distinct neuron populations in the medial geniculate body of the cat during classical conditioning. Behavioral Biology, 22, 275-301

StarR, A., \& Livingston, R. (1963), Long-lasting nervous system responses to prolonged sound stimulation in awake cats. Journal of Neurophysiology, 26, 416-431.

VAUghan, D. W. (1980). Thalamic and callosal connection of the rat auditory cortex. Brain Research, 260, 181-189.

WEINBERGER, N. M. (1982). Sensory plasticity and leaming: The magnocellular medial geniculate nucleus of the auditory system. In C. D. Woody (Ed.), Conditioning: Representation of involved neural function (pp. 697-710). New York: Plenum.

Weinberger, N. M., Ashe, J., Metherate, R., McKenna, T. M., DiAMOND, D. M., \& BAKIN, J. (1990). Retuning auditory cortex by learning: A preliminary model. Concepts in Neuroscience, 1, 91-132. Weinberger, N. M., Hopxins, W., \& Diamond, D. M. (1984). Physiological plasticity of single neurons in auditory cortex of the cat during acquisition of the pupillary conditioned response: Primary field (AI). Behavioral Neuroscience, 98, 171-188.

YounG, E. D., Brownell, W. E. (1976). Responses to tones and noise of single cells in dorsal cochlear nucleus of unanesthetized cats. Journal of Neurophysiology, 39, 282-300.

ZILLES, K. (1985). The cortex of the rat: A stereotaxic atlas. BerlinHeidelberg: Springer-Verlag.

(Manuscript received February 21, 1990; revision accepted for publication June 28,1990 .)

\title{
Erratum
}

Burton, P. G. A search for explanation of the brain and learning: Elements of the psychonomic interface between psychology and neurophysiology-II. Early behavior and its control, the origin of consciousness, and the rise of symbolic thought. Psychobiology, 1990, 18, 162-194The main heading on page 188

\section{BROAD-CONTEXT AND OJECTIVE REPRESENTATIONS}

should read

\author{
BROAD-CONTEXT AND \\ PROJECTIVE REPRESENTATIONS
}

